\title{
PRÁTICAS METODOLÓGICAS EM ADMINISTRAÇÃO: \\ O CASO DA UTILIZAÇÃO DA METODOLOGIA-Q COMO FERRAMENTA PARA PESQUISA E DIAGNÓSTICO DA CULTURA ORGANIZACIONAL
}

\author{
METHODOLOGICAL PRACTICES IN BUSINESS \\ ADMINISTRATION: THE USE OF Q-METHODOLOGY AS A \\ METHOD OF DIAGNOSING ORGANIZATIONAL CULTURE
}

\author{
Recebido em: 28/08/2012 Aprovado em: 05/12/2012 \\ Avaliado pelo sistema double blind review \\ Editora Científica: Manolita Correia Lima
}

\author{
PATRICIAAMÉLIA TOMEI patomei@iag.puc-rio.br \\ GIUSEPPE MARIA RUSSO \\ PONTIFÍCIA UNIVERSIDADE CATÓLICA DO RIO DE JANEIRO
}

\begin{abstract}
RESUMO
A busca por métodos de diagnóstico da cultura organizacional tem sido intensa nos últimos anos, uma vez que esta informação se tornou cada vez mais importante na definição de estratégias para a mudança. Várias metodologias qualitativas e quantitativas foram utilizadas na vasta literatura organizacional; no entanto, a Metodologia-Q (Brown, 1986) tem sido negligenciada. Este é o propósito deste artigo: utilizar a Metodologia-Q como um instrumento de análise da hierarquia de valores organizacionais, que servem de construto da cultura organizacional. Nesta pesquisa, com base em um estudo de caso de uma fusão de duas empresas multinacionais, foi considerada a diferença entre a cultura das organizações e as suas dificuldades de integração. Conclui-se que a Metodologia-Q proporcionou a oportunidade de investigar a subjetividade da cultura organizacional das empresas analisadas de forma sistemática, e que a flexibilidade de utilização deste método em profundidade para pequenas amostras pode contribuir na pesquisa exploratória com o mínimo de interferência do pesquisador.

Palavras-chave: cultura organizacional; valores organizacionais; metodologia-Q; Q-sort; fusão e aquisição.
\end{abstract}

\footnotetext{
ABSTRACT

The search for organizational culture diagnostic methods has been intense in recent years, since this information has become increasingly important in defining change strategies. A variety of qualitative and quantitative methodologies can be found from the extensive organizational literature available; however, the Q-methodology (Brown, 1986) has been neglected. Thus the purpose of this paper: to apply Q-Methodology as an instrument in the analysis of the organizational hierarchy of values that serve as the framework for organizational culture. This research, based on a case study of a merger between two multinational companies, considered the difference between the legacy of the organizations' cultures and their integration difficulties. It was concluded that the Q-methodology provided an opportunity to systematically investigate the subjectivity of the organizational culture of the companies analyzed, and that the flexibility of using it in depth for small samples can help in exploratory research with minimal interference from the researcher.

Keywords: organizational culture; organizational values; Q-methodology; Q-sort; merger and acquisition.
} 


\section{INTRODUÇÃO}

É consenso na vasta literatura organizacional que a cultura organizacional está relacionada ao processo de mudança, à implementação de estratégias e à obtenção de vantagens competitivas (TOMEI, 2008). Seguindo a linha de gerenciamento cultural, Tomei e Braunstein (1994) enfatizam que a cultura de uma organização é difícil de ser mudada, pois, como exige tempo para se formar e desenvolver, ela cria e mantém uma ordem, que passa por um processo lento, difícil e nem sempre bem-sucedido para ser modificada. No entanto, uma pergunta, que emerge destas dificuldades de gerenciamento, é se existe algum fator que auxilie ou facilite o gerenciamento da cultura organizacional ou se este é sempre algo demasiadamente complexo de fazer, haja vista a importância da cultura organizacional nos processos de mudanças.

Empresas bem-sucedidas sabem que os valores organizacionais, que servem de arcabouço da cultura, estão inseridos na estratégia organizacional e que exercem pressão sobre a tomada de decisão. Sem o entendimento destes valores, são grandes as chances de que uma mudança organizacional não tenha sucesso (TOMEI, 2008). Assim sendo, um aspecto essencial para se gerenciar a cultura de uma organização é compreendê-la, identificando com a maior precisão possível os valores da cultura organizacional existente para que, com o auxílio do gerenciamento cultural, seja possível determinar os valores que se busca manter e os que se pretende eliminar.

Schein (1996, p.I2) argumenta que "na prática, o estímulo para a mudança cultural é originado na necessidade de solucionar problemas organizacionais. Apenas quando premissas culturais seguem nesta direção é que a mudança cultural tem condições de nascer". Nesse sentido, Tomei e Braunstein (1994) apresentam quatro grupos de situações que são potenciais estímulos para o gerenciamento da cultura organizacional e a catalisação do processo de mudança. São elas: (I) fusões, aquisições, reestruturações, downsizings, terceirizações e privatizações; (2) fatores externos, como recessão, oportunidades tecnológicas e de mercado; (3) crises internas e 
processo de sucessão de poder; e, (4) processos de socialização de novos membros na organização.

No entanto, como identificar os valores organizacionais para que se possa gerenciar a cultura organizacional num processo de mudança? Diversas metodologias qualitativas e quantitativas, com o uso de variados métodos, têm sido utilizadas para realizar o diagnóstico da cultura organizacional (HOFSTEDE, I99I; SCHWARTZ, I994, I999; SCHNEIDER, I996; HANDY, I978; SETHIA; VON GLINOW, I985; QUINN; CAMERON, I983; TROMPENAARS, I993; BARROS; PRATES, I996; O’REILLY; CHATMAN; CALDWELL, I99I; SARROS et al., 2005).

Entretanto, acredita-se que outros métodos podem ser utilizados. Um método pouco explorado na literatura acadêmica para o diagnóstico cultural é a Metodologia-Q (BRown, 1986; Stephenson, 1953 apud santos; AMARAL, 2004, p. 6). Os principais motivos são a complexidade da aplicação e, principalmente, a análise dos dados resultantes do levantamento.

Buscando investigar e compreender melhor o método da Metodologia-Q, neste artigo utiliza-se uma situação de fusão organizacional, normalmente descrita como a consolidação de duas organizações em uma única. A escolha de uma fusão de duas multinacionais como estudo de caso para a aplicação da Metodologia-Q se deve ao fato de que entre $35 \%$ e $50 \%$ de todas as fusões fracassem por causa de problemas de recursos humanos e $85 \%$ dos fracassos são imputáveis à gestão da cultura organizacional (MITLETON-KELLY, 2006). É possível superar as dificuldades relacionadas com a fusão, uma vez que um elevado grau de integração cultural resulta do desejo de criar sinergias entre as duas empresas envolvidas (NAHAVANDIN; MALEKZADEH, I998; NGUYEN; KLEINER, 2003; MARKS; MIRVIS, I992).

Portanto o objetivo geral deste estudo é enriquecer a discussão metodológica utilizada no âmbito da investigação da cultura organizacional, analisando os resultados da aplicação da Metodologia-Q num estudo de caso.

Conforme se observa na literatura descrita a seguir, há uma necessidade de trabalhos neste sentido, já que as abordagens metodológicas para a análise da cultura organizacional, apesar de serem aceitas no âmbito 
dos estudos organizacionais, ainda apresentam pontos que precisam ser complementados, visando ao aprimoramento e à adaptabilidade à realidade organizacional, sem ferir os fundamentos básicos dos métodos e técnicas empregados.

Para tanto, este artigo está dividido em cinco partes. A primeira parte é essa introdução, já na segunda parte é apresentado a revisão da literatura, contendo: as definições sobre cultura organizacional, o papel dos valores organizacionais na cultura organizacional, a Metodologia-Q e o ocP (Organizational Cultural Profile) descrevendo a sua aplicação ao estudo de caso da fusão organizacional. Na terceira parte é apresentada a metodologia deste estudo. Na quarta os resultados e análises da aplicação metodológica, e, por fim, as considerações finais com as recomendações pertinentes. 
REVISÃO DA LITERATURA

\section{CULTURA ORGANIZATIONAL}

As definições que o termo cultura organizacional tem recebido apresentam diversos termos em comum, como costumes, valores, crenças e modo de agir, entre outras, que aparecem em grande parte das definições existentes. Katz e Kahn (I976, p.85) acreditam que "toda organização cria sua própria cultura ou clima, com seus próprios tabus, usos e costumes". Para os autores a cultura é originada tanto das normas e valores da organização formal como da sua reinterpretação pela organização informal.

Kilmann, Saxton e Serpa (1985, p.5) enfatizam que a "cultura pode ser definida como as filosofias, ideologias, valores, pressupostos, crenças, expectativas, atitudes e normas compartilhadas, que mantêm a comunidade unida”. Lorsch (1985 apud GORDON; DITOMASO, 1992) usa o termo cultura como as crenças compartilhadas entre gerentes e outros empregados. Estas crenças são frequentemente invisíveis, até para as próprias pessoas da organização, apesar de exercerem grande impacto sobre seus pensamentos e ações.

Um dos mais respeitados estudiosos da cultura organizacional, Edgar Schein (I992), apresenta o seguinte conceito para o termo:

Cultura organizacional é um padrão de pressupostos básicos compartilhados que um grupo aprendeu ao resolver seus problemas de adaptação externa e integração interna e que funcionou bem o suficiente para serem considerados válidos e ensinados a novos membros como a forma correta de perceber, pensar e sentir com relação a esses problemas (sCHein, I992, p.52).

O padrão de pressupostos básicos diz respeito a valores que regem o comportamento. Estes valores vão sendo internalizados e se tornando inconscientes a ponto de passarem a ser considerados naturais. Para Schein (I992), "a essência da cultura são esses valores, crenças e certezas aprendidos em conjunto, que são compartilhados e tidos como corretos” (sCHEIN, 200I, p.35). A abordagem apresentada por Schein (1992) mostra que o núcleo da cultura organizacional (certezas tácitas) é composto por valores que até 
os próprios indivíduos muitas vezes desconhecem. Decifrar os padrões culturais pode ajudar a prever ou explicar comportamentos.

O’Reilly, Chatman e Caldwell (199I) e Chatman e Jehn (1994) definem a cultura organizacional como um conjunto de valores compartilhados entre os membros da organização. Para Denison (1984), cultura organizacional são crenças e valores que tendem a ser manifestados nos comportamentos de seus membros. Similarmente, Eldridge e Crombie (1974, p. 89) definem a cultura organizacional como "uma configuração única das normas, dos valores, das crenças e comportamentos que caracterizam a maneira em que os grupos e os indivíduos combinam para realizar as coisas". Os valores foram também apresentados como motivadores da excelência organizacional (PETERS; WATERMAN, 1986). A cultura, para Mintzberg e Quinn (I99I), foi definida como um sistema bem desenvolvido e profundamente enraizado de valores. Para Chatman e Jehn (1994), cultura é representada pelos valores compartilhados entre os membros da organização e provê para esta última uma identidade.

As definições sobre cultura organizacional podem diferir em vários aspectos, mas tendem a um consenso com respeito ao fato de que os valores constituem um importante componente da cultura organizacional (мichilova; HUtCHINGS, 2006). Nesse sentido, valores organizacionais constituem os principais elementos de pesquisa da cultura organizacional (CHATMAN, I99I; O'Reilly; ChatMan; CALDWEll, I99I; SCHEIN, I996; VANDENBERGHE, 1999).

\section{O PAPEL DOS VALORES ORGANIZACIONAIS NA CULTURA}

Valores organizacionais estão entre os poucos conceitos sociopsicológicos, que têm sido adotados com sucesso por quase todas as disciplinas das ciências sociais e de gestão, e têm sido definidos como crenças organizacionais generalizáveis e duradouras sobre o desejo pessoal e social de modos de conduta (KLENKe, 2005). Para Klenke (2005), os valores são a base para a geração de comportamento, o qual se ajusta às necessidades dos grupos, onde diferentes valores têm graus de importância diferenciados para cada indivíduo. Nesse sentido, o autor enfatiza que valores são como construtos 
latentes envolvidos na avaliação de atividades ou resultados, como uma natureza geral e aplicável a vários níveis como nações, grupos e indivíduos. Por isso, os valores existem em redes organizadas e servem como guia regulatório, especificando, por exemplo, modos de comportamento que são socialmente aceitos. Assim, os valores quando compartilhados entre os membros das organizações compõem a base da cultura organizacional.

Para Klenke (2005), embora os valores sejam conceituados de várias maneiras, há determinadas qualidades que são comuns entre as definições existentes: (I) têm uma natureza mais geral do que específica; (2) podem ser aplicados em múltiplos níveis; (3) são duradouros e transcendem situações específicas; (4) provêem senso de propósito para o comportamento individual; e, (5) são a base para a geração de comportamentos adequados às necessidades dos grupos.

Segundo Michilova e Hutchings (2006) os valores têm sido tradicionalmente assunto do interesse e da análise de várias disciplinas. Os valores foram enfatizados por antropólogos como o núcleo da cultura. Os sociólogos enfatizaram sua natureza normativa e sua especificidade de serem compartilhados pela sociedade e pelos integrantes das organizações. Os psicólogos estudaram os valores associados com seu papel como determinantes do comportamento individual. A literatura organizacional e, particularmente, a literatura comportamental, enfatizou a importância dos valores como base para os membros das organizações compreenderem o mundo em torno deles e para agirem.

Os valores organizacionais foram definidos por Tamayo, Mendes e Paz (2000, p. 293) "como princípios ou crenças, organizados hierarquicamente, relativos a estados de existência ou a modelos de comportamentos desejáveis que orientam a vida da empresa”. Assim, valores são considerados como influenciadores substanciais em respostas atitudinais e comportamentais dos indivíduos (MEGLINO; RAVLIN, I998).

Para Deal e Kennedy (apud Freitas, I99I), valores constituem o núcleo da cultura. Para estes autores são os valores compartilhados, que definem o caráter fundamental da organização, aquilo que diferencia uma organização da outra. Para outros autores (CURRY; MOORE, 2003) é somente 
com os valores compartilhados que a parceria pode ter sucesso. A noção de compartilhamento, defendida por Freitas (199I), pressupõe que não há uniformidade e sim convivência com os valores da organização quando esses são diferentes dos valores individuais (TROMPENAARS, I993; HOFSTEDE, I99I; MORGAN, I996; PETTIGREW, I996).

O entendimento de valor como preferência permite inferir que os valores são reafirmados em comportamentos eficazes, são internalizados gradativamente e passam a ser pressupostos subjacentes da cultura organizacional (NOGUeIRA; MACHADo-DA-SILVA, 2003). A definição de valores apresenta a importância que esses têm para a determinação dos comportamentos. A relação entre valores e comportamento tem sido um dos principais objetivos de pesquisa na área de valores (BRITTO; PORTO; ANDRADE, 2003), indicando a importância dos valores para as organizações. Os valores são de grande importância para as organizações, tornando a sua compreensão relevante. Dessa forma, entende-se que os valores organizacionais são exclusivos em cada organização. Daft (2004) relata sobre os valores subjacentes e relaciona que os mesmos refletem os mais profundos processos de pensamento e são a verdadeira base da identificação e interpretação da cultura.

Embora a cultura organizacional não possa ser alterada rapidamente, é crítico para a nova organização compreender as bagagens culturais, que os funcionários trazem das suas empresas anteriores, a fim de criar uma nova cultura organizacional, que todos os membros estejam dispostos a apoiar.

\section{A METODOLOGIA-Q E O OPC (ORGANIZATIONAL CULTURAL PROFILE)}

Entendendo a cultura organizacional como um conjunto de valores compartilhados entre os membros da organização (HOwARD, 1998), e sabendo que existem diversas alternativas de conceituar a cultura organizacional, este estudo optou pela identificação da cultura organizacional pelo uso dos valores organizacionais, pela crença existente que eles direcionam os comportamentos das pessoas e por saber que a cultura essencial difere entre as organizações. 
O modelo de mapeamento da cultura organizacional proposto por O'Reilly, Chatman e Caldwell (199I), denominado de ocp (Organizational Cultural Profile), foi desenvolvido para examinar a congruência entre os valores individuais e organizacionais. Ele foi reduzido inicialmente por Cable e Judge (1996) e utilizado, posteriormente, num trabalho feito para confirmar a importância do ajuste da pessoa à organização no recrutamento e seleção de pessoal (CABLE; PARSONS, 200I). Os fatores do ocP refletiriam um padrão de relacionamento, baseado nos interesses individuais (por exemplo: apoio, ênfase no reconhecimento, orientação para equipes, decisão) e interesses organizacionais (por exemplo: inovação, atenção a detalhes, orientação para resultados, agressividade).

O ocp original é composto de 54 valores organizacionais, que surgiram de uma revisão feita por acadêmicos e escritores focados em valores e cultura organizacional. Trinta e oito chefes de departamento de escolas de negócio e todos os professores de quatro universidades de negócios dos Estados Unidos verificaram a existência de redundância, irrelevância e dificuldade de compreensão num conjunto inicial de iıo valores organizacionais. Após diversas interações, um conjunto final de 54 valores organizacionais foi utilizado empiricamente (O'REILLY; CHATMAN; CALDWELL, I99I).

O осP é baseado na técnica do Q-sort (вLOcK, 1978). Para aplicação do instrumento, como sugerido por O’Reilly, Chatman e Caldwell (199i), os respondentes deviam classificar os 54 valores do ocp em 9 categorias, que descrevessem o quanto cada valor era desejável na organização em que trabalhavam. Para a análise foi adotada a Metodologia-Q (a seguir detalhada).

A versão original do ocp foi desenvolvida usando a análise de fator exploratória para estabelecer oito dimensões da cultura organizacional: inovação, atenção ao detalhe, orientação para resultado, agressividade, apoio, ênfase em recompensas, orientação da equipe, e decisão. O’Reilly, Chatman e Caldwell (199I) nomearam os fatores que combinavam melhor às descrições na literatura de cultura e valores organizacional naquele tempo e que eram fáceis de compreender. Posteriormente, o ocp foi revisado e reduzido, recebendo também de diversos pequisadores a sugestão para 
estudos de validação. Vandenberghe (1999, p.I83) recomendou a aplicação do modelo que comparava nações e indústrias. Windsor e Ashkanasy (1996) sugeriram que os valores que compõe o ocp original deveriam ser avaliados em termos de sua adequação para amostras específicas. Também Howard (1998) sugeriu que a confiabilidade de todos os valores do ocp requer investigação. Outros pesquisadores como Vandenberghe (1999), Cable e Judge (1996) e Sarros et al. (2005) buscaram revisar e validar o ocp. Neste estudo utiliza-se esta revisão feita por Sarros at al. (2005) para mapear a cultura organizacional (o uso do construto do ocp revisado, utilizado nesta pesquisa, foi autorizado pelo James C. Sarros).

Sarros et al. (2005) realizaram uma pesquisa com 1918 executivos australianos estratificados pelo território do país. $\mathrm{Na}$ análise realizada por Sarros et al. (2005) com os 54 valores de O’Reilly, Chatman e Caldwell (I99I), foi possível verificar que diversos valores tiveram carregamentos negativos em seu fator correspondente. Estes valores foram removidos e outros valores tornaram-se redundantes ou apresentaram baixo Alfa de Crombach produzindo modelos inadequados e também foram excluídos. Entretanto, dois fatores novos foram identificados: responsabilidade social e estabilidade. Todos os cálculos foram validados, não apresentando diferença significativa entre as subamostras aleatórias selecionadas. Baseada na natureza dos valores carregados em cada fator, a nova versão do ocp reduzida apresenta 28 valores e sete fatores (Quadro I): Competitiveness, Social Responsibility, Supportiveness, Innovation, Emphasis on Rewards, Performance Orientation, Stability.

Quadro 1 Organization Cultural Profile - Factors and Values

\begin{tabular}{|c|c|}
\hline Factors & Values \\
\hline Competitiveness & $\begin{array}{l}\text { achievement orientation } \\
\text { emphasis on quality } \\
\text { being distinctive } \\
\text { being competitive }\end{array}$ \\
\hline Social Responsibility & $\begin{array}{l}\text { being reflective } \\
\text { having a good reputation } \\
\text { being socially responsible } \\
\text { having clear guiding philosophy }\end{array}$ \\
\hline
\end{tabular}




\begin{tabular}{|l|l|}
\hline Factors & Values \\
\hline Supportiveness & $\begin{array}{l}\text { being team oriented } \\
\text { sharing information freely } \\
\text { being people oriented } \\
\text { collaboration }\end{array}$ \\
\hline being innovative \\
quick to take advantage of opportunities \\
risk taking \\
taking individual responsibility \\
fairness \\
opportunities for professional growth \\
high pay for good performance \\
praise for good performance \\
having high expectations for performance \\
enthusiasm for the job \\
being results oriented \\
being highly organized, \\
stability \\
being calm \\
security of employment \\
low conflict
\end{tabular}

Fonte: Sarros et al. (2005).

Dado que os valores organizacionais são importantes (KABANOFF; DALY, 2002), como podem ser descritos e mensurados para se fazer comparações? Segundo Altman (1992), os estudos de valores organizacionais foram realizados adotando-se uma das seguintes metodologias: qualitativa, compreendendo uma ou várias organizações (por exemplo, sCHEIN, I992) e, quantitativa, tipo survey, envolvendo, em alguns casos, um grande número de organizações (por exemplo, chATMAN; JEHN, I994). Além disso, em conjunto, as metodologias quantitativa e qualitativa permitem a triangulação das pesquisas (FLICK, 2004). Para Flick (2004, p.274), a triangulação é entendida "como a compensação complementar das deficiências e dos pontos obscuros de cada metodologia isoladamente”. No nível empírico, há um grande interesse dos pesquisadores na integração dos métodos (DENISON; MISHRA, I995), demonstrando a importância das perspectivas funcionalista e fenomenológica (MALufe; GaTTI, 1987). Para Eisenhardt (I989), a combinação dos métodos pode ser muito sinérgica. As evidências quantitativas podem indicar relacionamentos, que podem não ser aparentes, e podem distanciar 
o pesquisador das impressões muitas vezes falsas dos dados quantitativos. Por outro lado, os dados qualitativos são muito importantes para entender o racional não revelado pelos dados quantitativos.

Assim, o resultado do estudo empírico sobre cultura organizacional de Denison e Mishra (1995) deu suporte para a importância do uso de métodos quantitativos e qualitativos, simultaneamente, apesar das críticas à utilização de questionários e à análise de organizações com o uso de modelos de categorização (SCHEIN, 200I). Entretanto, muitos pesquisadores apresentaram argumentos favoráveis à utilização de questionários e modelos de análise qualitativa da cultura organizacional (HANDY, 1978; HOFSTEDE, I99I; SCHNEIDER, I996; TROMPENAARS, I993; O'REILLY; CHATMAN; CALDWELL, I99I; SARROS et al., 2005).

A Metodologia-Q foi desenvolvida por William Stephenson em 1953 (BROWN, I986; SANTOS; AMARAL, 2004), tendo sido estudada extensivamente em muitos trabalhos que a referenciam (BRown, 1986). Basicamente, a Metodologia-Q proporciona fundamentos para o estudo sistemático da subjetividade. Esta característica torna-a adequada ao estudo de aspectos qualitativos relacionados com o comportamento humano. A forma de ordenação das variáveis utilizada na Metodologia-Q chama-se técnica Q-sort. A característica distintiva da técnica Q-sort requer que os participantes ordenem as variáveis fornecidas segundo uma distribuição predefinida, quase-normal. Na técnica Q-sort é raro trabalhar com painéis com mais de Ioo participantes, aos quais é pedido que ordenem listas de 20 a 50 variáveis (SANTOS; AMARAL, 2004).

A técnica Q-sort apresenta vários benefícios (THOMAs; WATSON, 2002): (I) É um meio de estudo em profundidade para pequenas amostras, (2) Pode ajudar na investigação exploratória, (3) Captura a subjetividade com a mínima interferência do investigador, (4) Os participantes da amostra não precisam ser selecionados aleatoriamente, (5) Pode ser aplicado pela Internet. A maior vantagem deste método em relação ao método tradicional (escala Likert) da atribuição de um valor às questões, é que o método induz os participantes a considerarem o problema como um todo, ao contrário do método tradicional que opera questão a questão. 
A opção da aplicação da técnica Q-sort em detrimento da escala Likert, opção normal nos estudos de pesquisa, deveu-se ao fato de o objetivo do estudo ser a ordenação de um conjunto de variáveis de acordo com a sua importância. A escala Likert tem o inconveniente de o respondente olhar para cada variável individualmente e não como um todo relacionado. Ao considerar cada variável isoladamente, ao tratar das variáveis mais importantes e as menos importantes, torna-se muito difícil ponderar a sua importância relativa de acordo com a escala, tendendo a atribuir valores extremos. Esse fato produz muitas repetições entre as variáveis, o que não é desejável, dado que o objetivo é produzir uma lista ordenada de acordo com as importâncias relativas das variáveis. Com a técnica Q-sort esse problema fica ultrapassado, uma vez que o participante tem que olhar para as variáveis como um todo, dividi-las em três grupos - as mais importantes, as menos importantes e as de valor neutro, de acordo com uma distribuição quasenormal predefinida. Além disso, o participante terá que se concentrar sobre cada uma das listas separadamente e escolher a variável mais importante ou menos importante das variáveis, conforme a lista em causa. Desta forma, consegue-se uma lista por ordem de importância, sem ambiguidades de classificação e sem nenhuma probabilidade de ter variáveis repetidas na mesma posição.

A técnica Q-sort fornece diversas vantagens para este tipo de pesquisa. Primeiramente, permite hierarquizações comparativas de um grande número de variáveis (HOWARD, I998), um benefício importante dado ser uma medida ipsative. Em segundo, a técnica Q-sort força a criação de uma distribuição estandardizada, aproximadamente normal (quasenormal), facilitando as inferências e comparações estatísticas com os grupos (HOwARD, 1998). Além disso, sendo que cada variável é comparada com cada uma das demais variáveis, o resultado da técnica Q-sort é um perfil realístico com variáveis disponibilizadas numa ordem que reflete a importância relativa de cada variável com relação às demais, referente à empresa. É realístico, tendo em vista que é esperado que algumas variáveis sejam mais importantes para uma empresa quando comparada com outras. 
Apesar de ser possível no uso da Metodologia-Q hierarquizar as variáveis sem uma distribuição forçada (qualquer número de variáveis por categoria), o presente método é superior porque obriga os respondentes a hierarquizarem as variáveis por importância, em cada empresa. Sem esta imposição, seria fácil para os respondentes ter uma grande quantidade de variáveis avaliadas nos seus limites extremos, como no caso adotado pela escala Likert. Sem isto, não teria sido possível nesta pesquisa identificar os valores importantes para cada uma das organizações.

A técnica Q-sort (BLOCK, 1978), que provê uma avaliação ideográfica dos valores da organização, permitindo comparação (COOPER-THOMAS; VIANEN; ANDERSON, 2004), na aplicação presencial permitiu uma forma de medir ipsative, reduzindo a oportunidade de o respondente dar uma resposta socialmente desejável (COOPER-THOMAS; VIANEN; ANDERSON, 2004).

A técnica Q-sort é útil para pequenas amostras (ROSENBAUM; OSTROM; KUNTZE, 2005), sendo de 30 até 50 respondentes o suficiente para investigar a opinião púbica (HAZARI, 2005), podendo ser utilizada até por sete respondentes (LEE; YU, 2004). 


\section{METODOLOGIA}

\section{A APLICAÇÃO DA METODOLOGIA-Q NUM CASO DE FUSÃO ORGANIZACIONAL DA EMPRESA X E EMPRESA Y}

Embora neste estudo tenha-se utilizado o método quantitativo e o qualitativo, a apresentação da análise focalizou apenas a pesquisa quantitativa e sua metodologia, para atender ao objetivo deste trabalho.

$\mathrm{Na}$ pesquisa quantitativa foi utilizado o questionário de Sarros et al. (2005), como instrumento da pesquisa, e a técnica Q-sort (BLOCK, I978) com os valores descritos por Sarros et al. (2005). Isto permitiu a avaliação dos valores organizacionais para pequenos grupos, seguida de entrevista semiestruturada com todos os respondentes.

A escolha das organizações foi feita por conveniência, por se tratar de uma fusão em nível global, que envolvia duas multinacionais. A amostra foi não probabilística, identificada pela diretoria de Recursos Humanos da empresa XY, totalizando Io2 entrevistas com 49 funcionários da empresa $\mathrm{X}$ e 53 da empresa Y, distribuídos segundo localidades e nível hierárquico detalhados na Tabela I.

Tabela 1 Amostra

\begin{tabular}{llllllll|}
\hline Country & Level & & & & \multicolumn{3}{c|}{ Total } \\
\hline Argentina & $\mathbf{2}$ & $\mathbf{3}$ & $\mathbf{4}$ & $\mathbf{5}$ & Others & Quant. & $\%$ \\
\hline Brazil & 0 & 0 & 5 & 3 & 10 & 18 & $18 \%$ \\
Colombia & 0 & 0 & 7 & 4 & 6 & 49 & $48 \%$ \\
\hline Mexico & 0 & 0 & 9 & 4 & 5 & 17 & $17 \%$ \\
Others & 0 & 0 & 0 & 0 & 0 & 18 & $18 \%$ \\
\hline Total & $\mathbf{1}$ & $\mathbf{5}$ & $\mathbf{4 4}$ & $\mathbf{1 5}$ & $\mathbf{3 7}$ & $\mathbf{1 0 2}$ & \\
\% & $\mathbf{1} \%$ & $\mathbf{5 \%}$ & $\mathbf{4 3 \%}$ & $\mathbf{1 5 \%}$ & $\mathbf{3 6 \%}$ & & $\mathbf{1 0 0 \%}$
\end{tabular}

Para coletar os dados primários da pesquisa foi utilizado um questionário contendo as variáveis de cultura organizacional de Sarros et al. (2005). Além 
do questionário foram feitas entrevistas de forma semiestruturada (MYERS; NEWMAN, 2007).

Nesta pesquisa a aplicação da técnica Q-sort ocorreu da seguinte maneira. Cada entrevistado recebeu um questionário contendo os valores do construto (estatisticamente considerada uma variável independente). Em seguida, foi solicitado a cada respondente que marcasse os 28 valores em sete colunas. As colunas hierarquizaram valores de "mais característico" a "menos característico" na organização, sendo a coluna central o neutro (HOWARD, 1998). Na primeira coluna da esquerda (- 3 ) o entrevistado devia colocar os valores que menos se apresentavam na empresa onde trabalhava e na primeira coluna da direita $(+3)$ os valores que mais se apresentavam na empresa onde trabalha.

A técnica Q-sort objetivou obter a imagem da opinião do entrevistado sobre o objeto considerado (MALUFE; GATTI, 1987), no caso desta pesquisa, os valores da empresa onde trabalhava. Para facilitar a análise estatística, o número de valores em cada coluna foi especificado de modo que formasse uma distribuição quase-normal. O número de valores, assim, formava uma curva normal, simétrica e unimodal (HOWARD, 1998).

Tendo em vista que o questionário e as entrevistas foram feitos no idioma inglês, não houve a necessidade de realizar a tradução do instrumento, que continha os valores organizacionais de Sarros et al. (2005). A validade dos questionários, fundamentada na teoria, foi um fator essencial para tornar possível o tratamento estatístico dos dados, de forma a avaliar a influência relativa e a significância dos diferentes critérios, assim como seus relacionamentos e implicações.

A coleta de dados ocorreu de forma presencial, com o uso de questionário impresso, seguido de entrevista. Os dados do questionário foram transcritos individualmente para os softwares utilizados nas análises. O preenchimento do questionário feito presencialmente, seguido de entrevista, contribuiu para evitar vieses potenciais do entrevistador e transmitir mais segurança aos respondentes que, no anonimato, puderam se sentir mais à vontade $\mathrm{e}$ expressar as opiniões com mais precisão (MALUFE; GATTI, I987). Outra grande vantagem da coleta presencial foi entrevistar todos os participantes. 
As respostas às perguntas sobre valores organizacionais foram tabuladas e tratadas utilizando-se procedimentos estatísticos simples e multivariados no SPSS I2.0 e no PCQ I.4I, tais como:

1. Médias.

2. Kruskal-Wallis H-test (HAIR; ANDERSON; TATHAM; BLACK, I998) com o objetivo de verificar a existência de diferenças entre empresas para o mesmo grupo de variáveis ou fatores culturais.

3. Correlação de Pearson para calcular e determinar a força do relacionamento entre os fatores do ocp de cada empresa. Coeficientes positivos indicam que existe um relacionamento direto: quando uma variável aumenta, a outra também aumenta. Coeficientes negativos indicam que existe um relacionamento inverso: quando uma variável aumenta a outra diminui (HAIR et al., 1998).

4. Metodologia-Q (Brown, 1986; Stephenson, 1953 apud Santos; AMARAL, 2004, p. 6) para identificar o agrupamento dos respondentes e hierarquizar comparativamente as variáveis. Os respondentes altamente correlacionados entre si podem ser considerados como semelhantes, de uma mesma família (cluster). O número de famílias é, consequentemente, empírico e completamente dependente de como os respondentes elaboraram a técnica Q-sort. A metodologia força a criação de uma distribuição estandardizada, aproximadamente normal (quase-normal), facilitando as inferências e comparações estatísticas com os grupos (HOWARD, 1998). Além disso, sendo que cada variável é comparada com cada uma das demais variáveis, o resultado é um perfil realístico com variáveis disponibilizadas numa ordem que reflete a importância relativa de cada variável com relação às demais, referente à empresa.

5. Correlação Canônica para determinar o nível congruência das culturas das empresas. O indicador de congruência é o coeficiente de determinação canônico. Esta técnica não foi localizada na análise de congruência da cultura organizacional apesar de já ter sido utilizada nas análises entre estratégia e cultura organizacional 
(BATES; AMUNDSON; SCHROEDER; MORRIs, 1995) e desempenho e cultura organizacional (LEE; YU, 2004).

Com o auxílio dessas análises, foram extraídos os resultados e as conclusões da pesquisa. Chatman (199I) define como cristalização da cultura de uma organização a sua homogeneidade. Isto foi examinado, identificando-se inicialmente os valores organizacionais (variáveis) e depois a congruência da cultura organizacional (NAZIR, 2005). O resultado estatístico é o coeficiente de determinação canônico, descrevendo o nível de congruência das empresas. $\mathrm{O} \mathrm{R}^{2}$ canônico deve ser maior que 0,30 (HAIR et al., 1998) para a existência de congruência entre as empresas.

\section{RESULTADOS E ANÁLISES}

A estatística descritiva, incluindo a média, foi calculada para as variáveis do ocP para o legado de cada empresa.

Os valores com as maiores diferenças, de acordo com a análise KruskalWallis, são estatisticamente significativas para I8 dos 28 valores (significância $<0,05$ ). Isso significa mais de $50 \%$ de todos os valores. Os valores com as maiores diferenças são: Foco na realização, Ênfase na qualidade, Ser reflexiva, Compartilha informações livremente, Foco em pessoas, Correr riscos, Entusiasmo para o trabalho, Estabilidade, Ser calma e Garantia de emprego.

A estatística descritiva, incluindo a média, foi calculada também para os fatores (consolidação dos valores) do ocp para a empresa $\mathrm{X}$ e $\mathrm{Y}$, respectivamente.

Segundo a análise Kruskal-Wallis as diferenças entre a empresa X e Y são estatisticamente significantes em cinco dos sete fatores (significância $<$ $0,05)$. Isso significa mais de $70 \%$ de todos os fatores. Os fatores com maiores diferenças são: Estabilidade, Apoio e Inovação.

Para calcular e determinar a intensidade das relações entre os fatores ocP foi utilizada a Matriz de Correlação de Pearson. Nas empresas X e Y existem correlações entre as variáveis (os coeficientes não são zero), mas elas são fracas (todas abaixo $+/-0,600$ ), algumas são positivas e outras negativas, mas só é possível generalizar as que são significantes ( $\mathrm{p}<0$, or ou $\mathrm{p}<0,05$ ). 
Individualmente, foi possível verificar que nas empresas X e Y nenhum dos valores tem uma correlação forte $(>+/-0,600)$.

Na essência, a Metodologia-Q determina quantos respondentes diferentes existentes. Respondentes que são altamente correlacionados um com o outro podem ser considerados como pertencentes a uma mesma família. Este estudo provê informação de quantas diferentes famílias (agrupamento ou clusters) existem. O número de agrupamentos (clusters) é, portanto, puramente empírico e totalmente dependente da forma como a correlação ocorre. Os agrupamentos indicam diferentes concepções relacionadas com a Metodologia-Q, com aquelas pessoas que partilhem uma concepção comum definindo o mesmo agrupamento. Agrupamentos com correlações acima de 0,600 (positiva ou negativa) podem ser considerados significantes. Todos os subgrupos têm pelo menos uma carga significante.

A Metodologia-Q, na apresentação dos resultados, descreve possíveis formas de agrupamentos (clusters) dos valores organizacionais. Os agrupamentos podem ser estatisticamente significantes ou não significantes. Neste estudo de caso, por exemplo, a Metodologia-Q resultou em nove agrupamentos para a empresa $\mathrm{X}$ (A até $\mathrm{I}$ ) e seis para a empresa $\mathrm{Y}$ ( $\mathrm{A}$ até $\mathrm{E}$ ). Os agrupamentos estatisticamente significantes, entre as duas empresas, como exemplo, estão descritos no Quadro 2. 
Quadro 2 Agrupamentos ${ }^{1}$ das Empresas X e Y

\begin{tabular}{|c|c|c|}
\hline Agrupamentos & Empresa X & Empresa $Y$ \\
\hline A & $\begin{array}{l}\text { Foco em resultados, objetivos e } \\
\text { desempenho }\end{array}$ & $\begin{array}{l}\text { Ênfase na qualidade, estabilidade, boa repu- } \\
\text { tação, ser organizada, responsabilidade } \\
\text { social e garantia de emprego }\end{array}$ \\
\hline B & $\begin{array}{l}\text { Boa reputação, oportunidade para } \\
\text { crescimento profissional e foco em } \\
\text { pessoas }\end{array}$ & $\begin{array}{l}\text { Compartilha informações livremente, jus- } \\
\text { tiça e maior remuneração para alto desem- } \\
\text { penho }\end{array}$ \\
\hline C & $\begin{array}{l}\text { Responsabilidade individual e } \\
\text { ambiente prazeroso (calma, baixo } \\
\text { nível de conflito, garantia de } \\
\text { emprego e estabilidade) }\end{array}$ & $\begin{array}{l}\text { Oportunidades para crescimento profissio- } \\
\text { nal, estabilidade e foco em resultados }\end{array}$ \\
\hline D & $\begin{array}{l}\text { Foco em objetivos, resultados e } \\
\text { equipes }\end{array}$ & $\begin{array}{l}\text { Boa reputação, ênfase na qualidade e foco } \\
\text { em resultados e objetivos }\end{array}$ \\
\hline
\end{tabular}

A interpretação da Metodologia-Q procede inicialmente em termos de pontuação, ao invés de cargas. A pontuação é uma espécie de média da pontuação dada a uma declaração específica por parte de todos os tipos, associada com o fator. Cada valor foi ponderado para cada grupo em três níveis: alta relevância positiva, alta relevância negativa e a média entre os dois primeiros.

O pano de fundo da Metodologia-Q é a fatoração dos respondentes e não das variáveis, portanto, a metodologia promove a opinião partilhada. Não é somente importante saber o que cada indivíduo pensa de cada valor, mas qual a importância de cada valor no conjunto dos indivíduos. Os instrumentos de pesquisa, que utilizam a escala Likert (HOFSTEDE, I99I; SCHWARTZ, I994, I999; SCHNEIDER, I996; SETHIA; VON GLINOW, 1985; QUINN; CAMERON, I983; trompenaARs, I993; barros; Prates, I996; SARros et al., 2005), não promovem esta informação, altamente relevante para a gestão da cultura organizacional.

A escala Likert leva em consideração os dados coletados, de vários indivíduos, e analisa-os em conjunto com as variáveis envolvidas, em contraste a Metodologia-Q, que os analisa de forma comparativa, considerando

\footnotetext{
1 Para determinar a importância da correlação, antes de ser considerada substancial, o erro padrão foi calculado. Isto foi obtido com a expressão $1 /(\mathrm{RQ}(\mathrm{N}))$, onde $\mathrm{N}$ é o número de variáveis $(\mathrm{N}=28)$ e RQé a raiz quadrada. Analisando a Matriz de Correlação Fatorial das empresas X e Y, foi possível perceber alguns relacionamentos (os coeficientes não eram zero), mas eles eram fracos (todos menores que +/- o,60o).
} 
diversas variáveis. Assim, conforme Quadro 2, os agrupamentos apresentam o resultado de percepção dos indivíduos agrupados por interesse nos valores organizacionais. Dessa forma, é possível identificar quais os indivíduos de cada agrupamento. Quanto maior o número de indivíduos em cada agrupamento, maior o alinhamento cultural.

Outro resultado importante se refere à identificação das subculturas. Cada agrupamento é considerado como um subgrupo. Analogamente, é possível identificar quais os valores prioritários para cada grupo de respondentes.

Em função destes resultados, a Metodologia-Q é ideal para que a pesquisa, onde o objetivo é a busca do alinhamento cultural, que pretende identificar tipos de indivíduos que respondem de forma semelhante a certo conjunto de variáveis. 


\section{CONSIDERAÇÕES FINAIS}

Apesar da existência de diversos métodos para o mapeamento da cultura organizacional (HOFSTEDE, I99I; SCHWARTZ, 1994, 1999; SCHNEIDER, 1996; HANDY, I978; SETHIA; VON GLINOW, I985; QUINN; CAMERON, I983; TROMPENAARS, I993; BARROS; PRATES, I996; O'REILLY; CHATMAN; CALDWELL, I99I; SARROS et al., 2005), o uso da Metodologia-Q, além de apresentar resultados que são consistentes com outros métodos, descortina a possibilidade de uma análise qualitativa com o uso do questionário. Nesse sentido, a Metodologia-Q é complementar às abordagens qualitativas e também alinhada aos métodos quantitativos estruturados. No aspecto qualitativo, isto ocorre devido à Metodologia-Q obrigar o respondente a olhar as variáveis como um todo, de forma comparativa e ordená-las de acordo com o grau de importância de cada uma delas, independentemente do número de variáveis (HOwARD, 1998). Assim, cada alteração do grau de importância de uma variável, impacta nas demais variáveis do construto. No que diz repeito ao aspecto quantitativo, a Metodologia-Q tangibiliza as respostas, que são pontuadas com pesos que refletem o grau de importância de cada variável. Isto permite que o resultado quantitaivo da pesquisa seja analisado com o suporte das técnicas estatísticas comparativas e, consequentemente, dos softwares estatísticos disponíveis no mercado, como o spss. Isto ocorre devido à distribuição da amostra ser aproximadamente normal. A comparação (COOPER-THOMAs; VIANEN; ANDERSON, 2004) da amostra possibilita extrair a subjetividade do tema cultura organizacional, tendo em vista que para as organizações existe uma importância relativa de cada variável (valores organizacionais) com relação às demais. Apesar de esta conclusão ser intuitiva, somente com a Matodologia-Q é possível vaidá-la.

Como valor agregado, a Metodologia-Q disponibilizou a oportunidade de investigar um tema subjetivo como a cultura organizacional, de forma sistemática, isto é, utilizando um instrumento de pesquisa estruturado e as técnicas estatísticas que garantem significância ao resultado estatístico, transformando-se num método adequado ao estudo de aspectos qualitativos relacionados com o comportamento humano. 
Pela flexibilidade do uso deste método em profundidade para pequenas amostras, a Metodologia-Q pode ajudar na investigação exploratória com a mínima interferência do investigador. Isto é facilitado porque a metodologia permite inferências e comparações estatísticas com os grupos, colocando as variáveis numa ordem que reflete a importância relativa de cada variável com relação às demais, referente a cada empresa, e demonstrando as diferenças de importância quando comparada com outras empresas.

Como conclusão dos aspectos mencionados, pode-se afirmar que a Metologia-Q é um método que identifica a subjetividade das inter-relações existentes e semelhanças entre os indivíduos da amostra. Uma das principais diferenças entre o uso da escala Likert e a Metodologia-Q, do ponto de vista técnico, é que, o primeiro apresenta como resultado a média de cada item da escala (técnica dos componentes principais), de acordo com a escolha aleatória dos indivíduos da amostra, e o segundo a comparação de todas as variáveis e o agrupamento dos indivíduos por grau de importância (técnica do centróide). No primeiro caso, pode ocorrer com facilidade um viés nas respostas, caso todos os indivíduos da amostra selecionem todos os valores como muito presentes, ou inversamente, todos os valores como pouco presentes. Na Metodologia-Q isto não é possível, devido à técnica de escolha forçada adotada.

Por fim, vale lembrar que a Metodologia-Q não é um método estatístico no sentido empregado na Análise de Fator de redução de dados aplicado com a escala Likert, que busca identificar e combinar conjuntos de variáveis dependentes para mensurar coisas semelhantes, mas sim um método que busca identificar a subjetividade da cultura organizacional.

É muito importante selecionar qualquer metodologia reconhecendo as suas limitações. A Metodologia-Q obriga o respondente a pensar cuidadosamente sobre, por exemplo, quais valores organizacionais são os mais importantes para ele. É possível que o respondente nunca tenha priorizado os seus pensamentos e tomado decisões sobre o tema, neste estudo de caso, os valores organizacionais. Consequentemente, isso pode não ser fácil para algumas pessoas. Uma segunda limitação é que as escolhas representam uma tomada de decisão em um determinado momento, e 
podem mudar com o tempo ou sob diferentes circunstâncias. Finalmente, não se podem remover completamente as construções sociais do processo da pesquisa. Em outras palavras, o pesquisador nunca está ausente do processo de coleta de dados, e por isso, é possível que alguns indivíduos tomem suas decisões em função do que pensam que deveriam responder, com base numa perspectiva organizacional (coletiva), ao invés de individual. 


\section{REFERÊNCIAS}

ALTMAN, Y. Towards a Cultural Typology of European Work Values and Work Organization. Innovation in Social Sciences Research, v.5, n.1, 1992.

BARROS, B.T. de; PRATES, M.A.S. O estilo brasileiro de administrar. São Paulo: Atlas, 1996.

BATES, K.; AMUNDSON, S.; MORRIS, W.; SCHROEDER, R. The Crucial Interrelationship Between Manufacturing Strategy and Organizational Culture. Management Science, v.41, n.10, 1995.

BLOCK, J. The Q-sort Method in Personality Assessment and Psychiatric Research. Palo Alto: Consulting Psychologists Press, 1978.

BRITTO, M.J.P. de; PORTO, J.B.; ANDRADE, J.E.B. Valores, Suporte Psicossocial e Impacto do Treinamento no Trabalho. In: ENCONTRO DA ASSOCIAÇÃO NACIONAL DE PÓS-GRADUAÇÃO E PESQUISA EM ADMINISTRAÇÃO, 27., Atibaia (SP). Anais... 2003, Atibaia (SP).

BROWN, S.R., Q-technique and method. In: W.D.A.L.-B. Berry, M.S. (Eds) New tools for Social Scientists. Beverly Hills, CA: Sage, 1986.

CABLE D.M.; JUDGE, T.A. Person-organization Fit, Job Choice Decisions, and Organizational Entry. Organizational Behavior and Human Decision Processes, v.67, n.3, p.294-311, 1996.

CABLE D.M.; PARSONS, C.K. Socialization Tactics and Person-organization Fit. Personnel Psychology, v.54, p.1-23, 2001.

CHATMAN, J.A. Matching People and Organizations: selection and socialization in public accounting firms. Administrative Science Quarterly, v.36, p.459-484, 1991.

CHATMAN, J.A.; JEHN, K. Assessing the Relationship between Industry Characteristics and Organizational Culture: How Different Can You Be? Academy of Management Journal, v.37, p.522-553, 1994.

COOPER-THOMAS, H.D.; VIANEN, A.V.; ANDERSON, N. Changes in personorganization fit: The impact of socialization tactics on perceived and actual P-O fit. European Journal of Work and Organizational Psychology, v.13, n.1, p.52-78, 2004.

CURRY, A.; MOORE, C. Assessing information culture: an exploratory model. International. Journal of Information Management, v.23, n.2, p.91-110, 2003.

DAFT, R.L. Organization Theory \& Design, 8th ed. Thomson: South Western, 2004.

DENISON, D.R. Corporate Culture and Organizational Culture and Effectiveness. Organization Science, v.6, n.2, p.204-223, 1984.

DENISON, D.R.; MISHRA, A.K. Toward a theory of organizational culture and effectiveness. Organization Science, v.6, n.2, p.204-223, 1995. 
EISENHARDT, K.M. Building Theories from Case Study Research. Academy of Management Review, v.14, n.4, p.532-550, 1989.

ELDRIDGE, J.; CROMBIE, A. Sociology of Organizations. London: George Allen and Unwin, 1974.

FLICK, U. Uma Introdução à Pesquisa Qualitativa. 2. ed, Porto Alegre: Bookman, 2004.

FREITAS, M.E. de. Cultura organizacional: formação, tipologias e impactos. São Paulo: Makron, 1991.

GORDON, G.G.; DITOMASO, N. Predicting Corporate Performance from Organizational Culture. Journal of Management Studies, v.29, n.6, p.783-797, 1992.

HAIR JR., J.F., ANDERSON, R.E.; TATHAM, R.L.; BLACK, W.C. Multivariate Data Analysis. New Jersey: Prentice Hall, 1998.

HANDY, C.B. Como Compreender as Organizações. Rio de Janeiro: Zahar, 1978.

HAZARI, S. Perceptions of End-Users on the Requirements in Personal Firewall Software: An Exploratory Study. Journal of Organizational and End User Computing, v.17, n.3, p.47-65, 2005.

HOFSTEDE, G. Cultures and Organizations. Software of the Mind. Cambridge, Great Britain: McGraw-Hill Book Company, 1991.

HOWARD, L.W. Validating the Competing Values Model as a Representation of Organizational Cultures. The International Journal of Organizational Analysis, v.6, n.3, p.231-250, 1998.

KABANOFF, B.; DALY, J. Espoused values of organizations. Australian Journal of Management, v.27, 2002.

KATZ, D.; KAHN, R.L. Psicologia Social das Organizações. 2. ed. São Paulo: Atlas, 1976.

KILMANN, R.H.; SAXTON, M.J.; SERPA, R. Five key issues in understanding and changing culture. In: . Gaining control of the corporate culture. San Francisco: Jossey-Bass, 1985.

KLENKE, K. Corporate values as multi-level, multi-domain antecedents of leader behaviors. International Journal of Manpower. v.26, n.1; p.50-68, 2005.

LEE, S.K.J.; YU, K. Corporate Culture and Organizational Performance. Journal of Managerial Psychology, v.19, n.4, 2004.

MALUFE, J.R.; GATTI, B.A. Métodos de Pesquisa nas Relações Sociais. 2. ed. Volume 2: Medidas na Pesquisa Social. São Paulo: Editora Pedagógica e Universitária, 1987.

MARKS, M.L.; MIRVIS, P.H. Rebuilding After the Merger: dealing with survivor sickness. Organizational Dynamics, v.21, n.2, p.18-35, 1992.

MEGLINO, B.M.; RAVLIN, E.C. Individual values in organizations: concepts, controversies and research. Journal of Management, v.24, n.3, p.295-330, 1998. 
MICHAILOVA, S.; HUTCHINGS, K. National Cultural Influences on Knowledge Sharing: A Comparison of China and Russia. Journal of Management Studies, v.43, n.3, p.383-405, 2006.

MINTZBERG, H.; QUINN, J. The Strategy Process: Concepts, Contexts, Cases. 2. ed., Prentice-Hall, 1991.

MITLETON-KELLY, E. Coevolutionary integration: the co-creation of a new organization form following a merger and acquisition. Coevolutionary integration, v.8, n.2, 2006 .

MORGAN, G. Imagens da Organização. São Paulo: Atlas, 1996.

MYERS, M.D.; NEWMAN, M. The qualitative interview in IS research: Examining the craft. Information and Organization, v.7, p.2-26, 2007.

NAHAVANDIN, A.; MALEKZADEH, A. Acculturation in Mergers and Acquisitions. Academy of Management Review, v.13, n.1, 1998.

NAZIR, N.A. Person-Culture Fit and Employee Commitment in Banks. The Journal for Decision Makers, v.30, n.3, p.39-51, 2005.

NGUYEN, H.; KLEINER, B.H. The Effective Management of Mergers. Leadership \& Organization Development Journal, v.24, n.7/8, p.447-454, 2003.

NOGUEIRA, E.E.S.; MACHADO-DA-SILVA, C.L. Identidade Organizacional - a Importância dos Valores e Crenças: Estudo de Caso em uma Organização Extinta por Incorporação. In: ENCONTRO NACIONAL DE PÓS-GRADUAÇÃO e PESQUISA em ADMINISTRAÇÃO, 27., 2003, Atibaia (SP). Anais...

O’REILLY III, C.; CHATMAN, J.; CALDWELL, D. People and Organizational Culture: a Profile Comparison Approach to Assessing Person-Organization Fit. Academy of Management Journal, v.34, p. 487-516, 1991.

PETERS, T.J.; WATERMAN Jr., R.H. Vencendo a Crise. São Paulo: Harbra, 1986.

PETTIGREW, A.M. A Cultura das Organizações é Administrável? In: FLEURY, M.T.L.; FISCHER, R.M. (Org). Cultura e Poder nas Organizações. 2. ed. São Paulo: Atlas, 1996.

QUINN, R.E.; CAMERON, K. Organization Life Cycles and Shifting Criteria of Effectiveness: Some preliminary evidence. Management Science, v.29, n.1, p.33-51, 1983.

ROSENBAUM, M.S.; OSTROM, A.L.; KUNTZE, R. Loyalty programs and a sense of community. The Journal of Services Marketing, v.9, n.4, p.222-34, 2005.

SANTOS, L.D. dos; AMARAL, L. Estudos Delphi com Q-Sort sobre a web: a sua utilização em sistemas de informação. In: CONFERÊNCIA DA ASSOCIAÇÃO PORTUGUESA DE SISTEMAS DE INFORMAÇÃO, 5., Lisboa, 2004 - CAPSI 2004: actas da $5^{\text {a }}$ conferência. Disponível em CD-ROM.

SARROS, J.C.; GRAY, J.; DENSTEN, I.L.; COOPER, B. The Organizational Culture Profile Revisited and Revised: An Australian Perspective. Australian Journal of Management, v.30, n.1, p.159-182, 2005. 
SCHEIN, E.H. Organizational Culture and Leadership. 2.ed. San Francisco: Jossey-Bass, 1992.

SCHEIN, E.H. Three Cultures of Management. The Key to Organizational Learning. Sloan Management Review, v.38, n.1, p.9-20, 1996.

SCHEIN, E.H. Guia de Sobrevivência da Cultura Corporativa. Rio de Janeiro: José Olympio, 2001.

SCHNEIDER, W.E. Uma Alternativa à Reengenharia: um plano para fazer a cultura atual da sua empresa funcionar. Rio de Janeiro: Record, 1996.

SCHWARTZ, S.H. A Theory of Cultural Values and Some Implications for Work. Applied Psychology: an International Review, v.48, n.1, p.23-47, 1999.

SCHWARTZ, S.H. Beyond Individualism/Collectivism: New cultural dimensions of values. In: KIM, U.; TRIANDIS, H.C.; KAGITCIBASI, C.; CHOI, S.C.; YOON, G. Individualism and Collectivism: Theory, methods, and applications. Thousand Oaks: Sage, 1994.

SETHIA, N.; VON GLINOW, M.A. Arriving at Four Cultures by Managing the Reward System. In: KILMANN, R.H.; SAXTON, M.J.; SERPA, R. Gaining Control of the Corporate Culture. San Francisco: Jossey-Bass, 1985.

TAMAYO, A.; MENDES, A. M.; PAZ, M.G.T. Inventário de Valores Organizacionais. Estudos de Psicologia, v.5, n.2, p.289-315, 2000.

THOMAS, D.M.; WATSON, R.T. Q-Sorting and MIS Research: A Primer.

Communications of the Association for Information Systems, v.8, p.141-156, 2002.

TOMEI, P.A. Cultura e Mudanca Organizacional (org.), Editora PUC-Rio, Rio Janeiro, 2008

TOMEI, P. A.; BRAUNSTEIN, M. L.. Cultura Organizacional e Privatização-A Dimensão Humana. Makron Books, 1994.

TROMPENAARS, F. Riding the Waves of Culture: Understanding Cultural Diversity in Business. London: The Economist Press, 1993.

VANDENBERGHE, C. Organizational culture, person-culture fit, and turnover: A replication in the health care industry. Journal of Organizational Behavior, v.20, n.2, p.175184, 1999.

WINDSOR, C.A.; ASHKANASY, N.M. Auditor Independence Decision Making: The Role of Organization Culture Perceptions. Behavioral Research in Accounting, v.8, p.80-97, 1996. 


\section{DADOS DOS AUTORES}

\section{PATRICIA AMÉLIA TOMEI^ patomei@iag.puc-rio.br}

Doutora em Administração pela USP

Instituição de vinculação: Pontifícia Universidade Católica do Rio de Janeiro

Rio de Janeiro/RJ - Brasil

Áreas de interesse em pesquisa: Comportamento organizacional, Cultura, Poder e Mudança organizacional.

* Rua Marques de São Vicente, 225 Gávea Rio de Janeiro/RJ 22451-900

\section{GIUSEPPE MARIA RUSSO giuseppe.russo@globo.com}

Doutor em Administração pela Puc/RJ

Instituição de vinculação: Pontifícia Universidade Católica do Rio de Janeiro Rio de Janeiro/RJ - Brasil

Áreas de interesse em pesquisa: Cultura Organizacional e Remuneração. 